\title{
Population-level analysis reveals the widespread occurrence and phenotypic consequence of DNA methylation variation not tagged by genetic variation in maize
}

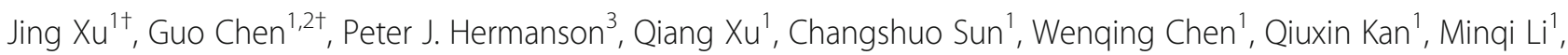
Peter A. Crisp ${ }^{3}$, Jianbing Yan ${ }^{1}$, Lin $\mathrm{Li}^{1}$, Nathan M. Springer ${ }^{3^{*}}$ and Qing $\mathrm{Li}^{i^{*}}$ (D)

\begin{abstract}
Background: DNA methylation can provide a source of heritable information that is sometimes entirely uncoupled from genetic variation. However, the extent of this uncoupling and the roles of DNA methylation in shaping diversity of both gene expression and phenotypes are hotly debated. Here, we investigate the genetic basis and biological functions of DNA methylation at a population scale in maize.

Results: We perform targeted DNA methylation profiling for a diverse panel of 263 maize inbred genotypes. All genotypes show similar levels of DNA methylation globally, highlighting the importance of DNA methylation in maize development. Nevertheless, we identify more than 16,000 differentially methylated regions (DMRs) that are distributed across the 10 maize chromosomes. Genome-wide association analysis with high-density genetic markers reveals that over $60 \%$ of the DMRs are not tagged by SNPs, suggesting the presence of unique information in DMRs. Strong associations between DMRs and the expression of many genes are identified in both the leaf and kernel tissues, pointing to the biological significance of methylation variation. Association analysis with 986 metabolic traits suggests that DNA methylation is associated with phenotypic variation of 156 traits. There are some traits that only show significant associations with DMRs and not with SNPs.
\end{abstract}

Conclusions: These results suggest that DNA methylation can provide unique information to explain phenotypic variation in maize.

Keywords: DNA methylation, Gene expression, Phenotypic diversity, Maize

\section{Background}

DNA methylation is the most studied chromatin modification in many plant species. DNA methylation has important roles in maintaining genome integrity and may also influence plant development and environmental responses, especially in species with complex genomes [1-6]. DNA methylation occurs in three sequence contexts in plants, CG, CHG, and $\mathrm{CHH}(\mathrm{H}=\mathrm{A}, \mathrm{C}$, or $\mathrm{T})$, each of which is

\footnotetext{
* Correspondence: springer@umn.edu; qingli@mail.hzau.edu.cn

${ }^{+}$Jing Xu and Guo Chen contributed equally to this work.

${ }^{3}$ Department of Plant and Microbial Biology, University of Minnesota, St. Paul, MN 55108, USA

${ }^{1}$ National Key Laboratory of Crop Genetic Improvement, Huazhong

Agricultural University, Wuhan 430070, China

Full list of author information is available at the end of the article
}

maintained by different pathways. CG is maintained by DNA METHYLTRANSFERASE 1 (MET1), CHG by CHROMOMETHYLASE 3 (CMT3), and CHH by RNA directed DNA Methylation (RdDM) as well as CHROMOMETHYLASE 2 (CMT2) $[2,7,8]$.

DNA methylation often varies across different individuals of the same species [9-13]. This can include spontaneous epimutations [9-11] as well as differences among genetically distinct varieties. Natural variation for DNA methylation includes examples in which genetic changes such as transposon insertions or rearrangements cause the change in methylation (obligatory epialleles) [14, 15]. There can also be more complex examples in which a genetic change results in a facilitated epiallele [14]. Other

(C) The Author(s). 2019 Open Access This article is distributed under the terms of the Creative Commons Attribution 4.0 International License (http://creativecommons.org/licenses/by/4.0/), which permits unrestricted use, distribution, and 
examples of natural variation for DNA methylation can reflect pure epigenetic variation that occurs in the absence of any causative genetic changes $[1,14]$. The association between DNA methylation and genetic variation has been reported at a genome-wide scale in Arabidopsis [12, 16, 17]. These studies suggest that some examples of variation for DNA methylation are due to genetic changes while others are not. In maize, a species with a larger genome and much higher transposon content, there is also evidence of both genetic-dependent and genetic-independent DNA methylation $[18,19]$. The previous studies in maize were not able to detect context-specific DNA methylation patterns, which may have different functions and stabilities.

DNA methylation can result in phenotypic changes, most likely through influencing gene expression. DNA methylation within a gene can affect splicing of premRNAs [20, 21]. DNA methylation outside gene, especially those within promoter regions, has been suggested to influence gene expression levels [22]. Several studies in plant species suggest that the number of genes whose expression is affected by DNA methylation is limited, at an order of hundreds, and the genes whose expression is affected by DNA methylation usually have specific properties [13, 23]. These genes generally show qualitative changes (on versus off) in expression among different genotypes [13]. However, these studies assayed gene expression in one tissue. It is possible that more genes for which expression are associated with DNA methylation can be identified if more tissues were assayed, as has been found for genetic variation [24-27].

In this study, we investigated the genetic basis and biological consequences of natural variation in DNA methylation among diverse inbred lines in maize. We found evidence for many examples of differentially methylated regions (DMRs) that contain information that is not fully captured using SNPs. We showed that variation in DNA methylation is associated with variation in gene expression, and this association is dependent upon sequence contexts as well as the position of DMR relative to gene transcriptional start site. Furthermore, we showed that variation in DNA methylation is associated with phenotypic variation in maize and can explain a portion of the heritability of some metabolic traits.

\section{Results}

\section{Extensive variation in DNA methylation among maize inbred lines}

To explore natural variation in DNA methylation, we profiled DNA methylation across a panel of 263 diverse maize inbred lines using a capture-based method [28]. This method allows single-base resolution of DNA methylation across a large population at a common set of loci with high coverage. The capture space includes over 20,000 regions, covering $15 \mathrm{Mb}$ of the maize genome [29]. These regions were selected based on our previous work of DNA methylation in maize and include regions that vary in DNA methylation across three maize lines or five maize tissues, $\mathrm{mCHH}$ islands, and promoters of genes that are potentially silenced by DNA methylation $[13,30]$. The maize inbreds utilized in this study represent a wide range of diversity, including lines that are adapted to tropical/semitropical regions or temperate regions. It includes the inbred B73 used to make the maize reference genome as well as inbreds widely used in past and present breeding schemes (Additional file 2: Table S1). We obtained a total of $\sim 2.3$ billion $2 \times 125$ paired reads, with an average of $\sim 8.7$ million paired reads for each sample (Additional file 2: Table S1). These reads were mapped to the B73 genome [31] at a mapping rate from 16 to $70 \%$, corresponding to an average of $\sim 3.8$ million mapped reads for each sample. At this mapping rate, we have a total of $\sim 7 \mathrm{Mb}$ of regions with $\geq 2$-fold coverage in $>60 \%$ of the lines, allowing us to identify regions with variable DNA methylation at a population scale in maize.

DMRs were identified for each of the three sequence contexts. In total, we identified 8864, 9759, and 5075 DMRs for CG, CHG, and CHH respectively. To support the reliability of the capture-based assay of DNA methylation, we compared DMRs that are identified using this method with the DMRs that are identified based on whole genome bisulfite sequencing (WGBS) [13]. We took advantage of B73 and Mo17 for which DNA methylation levels have been assayed using both methods. As expected, the DMRs that are identified using the capture-based method are well supported by WGBS data (Additional file 1: Figure S1). The DMRs were distributed along the 10 maize chromosomes. Many of the DMRs were located in regions annotated as genes, corresponding well with the distribution of capture probes (Fig. 1a, Additional file 1: Figure S2). The size of DMRs is quite similar for all three sequence contexts, with the majority between $60 \mathrm{bp}$ and $1000 \mathrm{bp}$ and a median of $200 \mathrm{bp}$ (Additional file 1: Figure S2a). This suggests that variation in DNA methylation is usually confined to a region corresponding to one or several nucleosomes. The analysis of the minor epiallele frequency (MEF) suggests that the lowly methylated state is often the rare epiallele for CG and CHG DMRs while CHH DMRs often exhibit higher methylated levels as the rare epiallele (Additional file 1: Figure S2b). The distribution of CG and CHG DMRs relative to genes and TEs is similar to the distribution of all assayed regions. The $\mathrm{CHH}$ DMRs are depleted within genes (Additional file 1: Figure S2c). Prior studies in Arabidopsis have identified natural variation in DNA methylation components such as VIM or CMT2 $[17,32,33]$ that influence genome-wide methylation levels or patterns in some ecotypes, but we did not find any evidence for this in maize. 

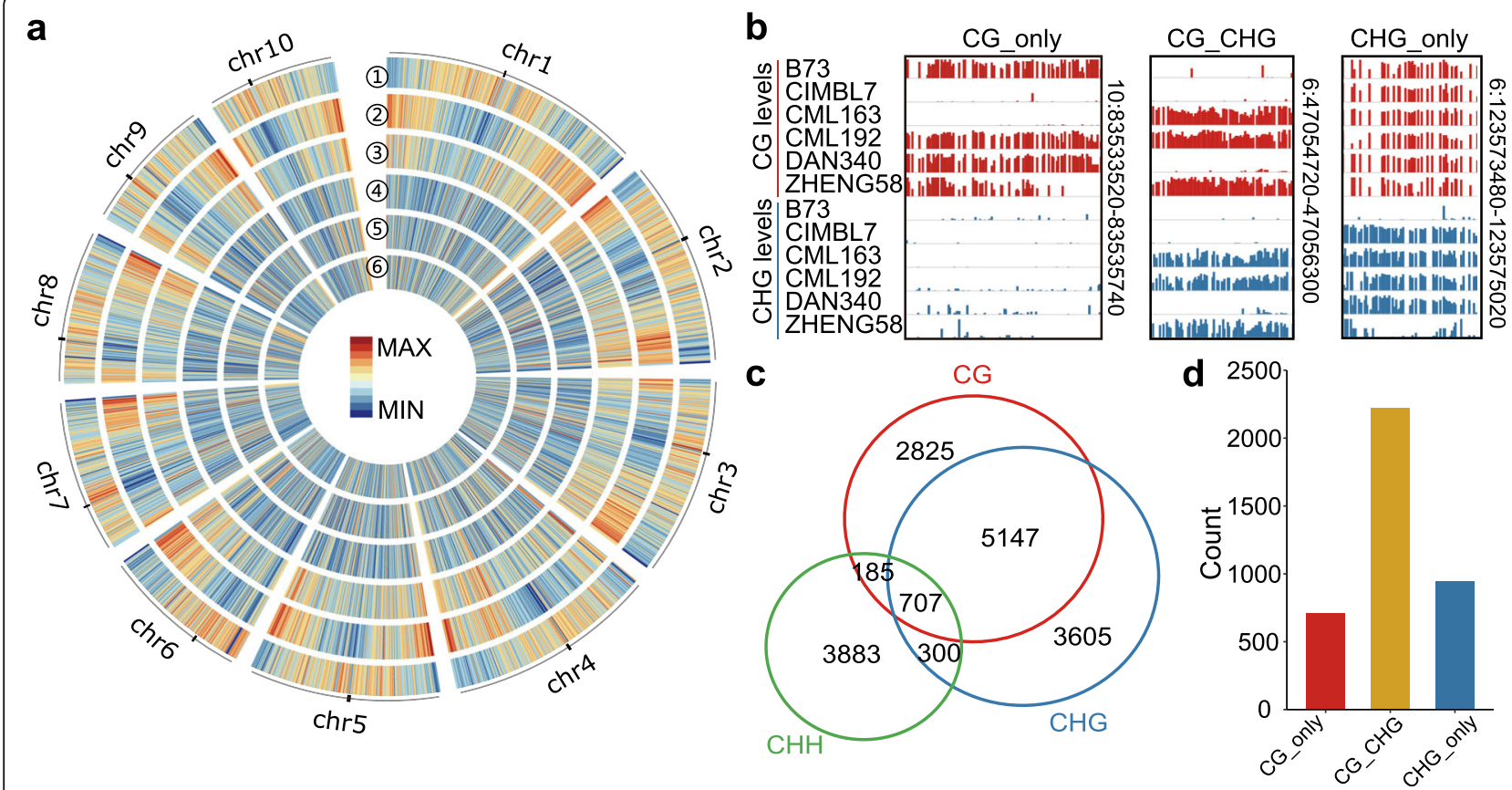

Fig. 1 Natural variation of DNA methylation among maize inbred lines. a The distribution of DMRs throughout maize chromosomes and the relationship with other genomic features is visualized. From the outer ring to the inner ring (1-6) are TEs, genes, regions with capture probes, CG DMRs, CHG DMRs, and CHH DMRs. Density of each type was calculated based on $1 \mathrm{Mb}$ windows, and the centromeres are represented by black blocks. b Examples of context-specific DMRs. Each track represents different inbred lines. c Overlaps between CG, CHG, and CHH DMRs. d Total number of context-specific DMRs

Though we identified DMRs separately for each context, there are many loci at which they can co-vary (Fig. 1b). In fact, there is substantial redundancy for DMRs for different contexts, especially for CG and CHG DMRs (Fig. 1c, d). We thus combined CG and CHG DMRs, and identified a set of regions that show variations in both contexts or only in CG or CHG context. A stringent set of criteria were applied (the "Methods" section) to identify 708 DMRs that vary only in CG context (CG_only), 944 DMRs that vary only in CHG context (CHG_only), and 2223 DMRs that vary in both CG and CHG contexts (CG_CHG). The relative frequency of context-specific DMRs within the captured loci is quite similar to the genome-wide patterns [13]. The size range of the context-specific DMRs is quite similar, but the overlap with genomic features is distinct (Additional file 1: Figure S2d, e).

\section{DMRs can differentiate maize subgroups}

We explored whether variation in DNA methylation accurately reflects genetic relationships among different inbred lines. Individual relatedness that is computed using either SNP or DNA methylation levels is highly correlated (Fig. 2a). Interestingly, the correlation is substantially higher for CG methylation at CG DMRs than for CHG (CHG DMRs) and $\mathrm{CHH}$ (CHH DMRs) methylation (Fig. 2b), suggesting that CG methylation is more stable. Principle component analysis (PCA) performed using CG, CHG, or CHH methylation levels from DMRs can separate inbreds into different subgroups, which agree well with classifications based on SNPs (Additional file 1: Figure S3a). The ability to differentiate population subgroups is highest for CG methylation (Additional file 1: Figure S3a). We then asked whether variable levels of CG (or CHG) methylation from CG_ only (or CHG_only) DMRs have similar power as that from CG_CHG DMRs to differentiate populations (Fig. 2c). Interestingly, the CG_only and CHG_only DMRs show less correlation with genetic distance than CG_CHG DMRs. The differentiation of population subgroups using DMRs suggests the presence of subgroupspecific DMRs. Indeed, an analysis of variance analysis (ANOVA) suggests that there are numerous DMRs that show high or low methylation in the majority of lines of a subgroup, though no DMRs that have exclusively low or high methylation in one subgroup were identified (Additional file 1: Figure S4).

\section{Genome-wide association analysis to dissect the genetic basis of DMRs}

DNA methylation could be associated with nearby genetic variation either due to the causal action of the genetic variation upon methylation levels or due to linkage disequilibrium (LD) between highly stable DMRs and 

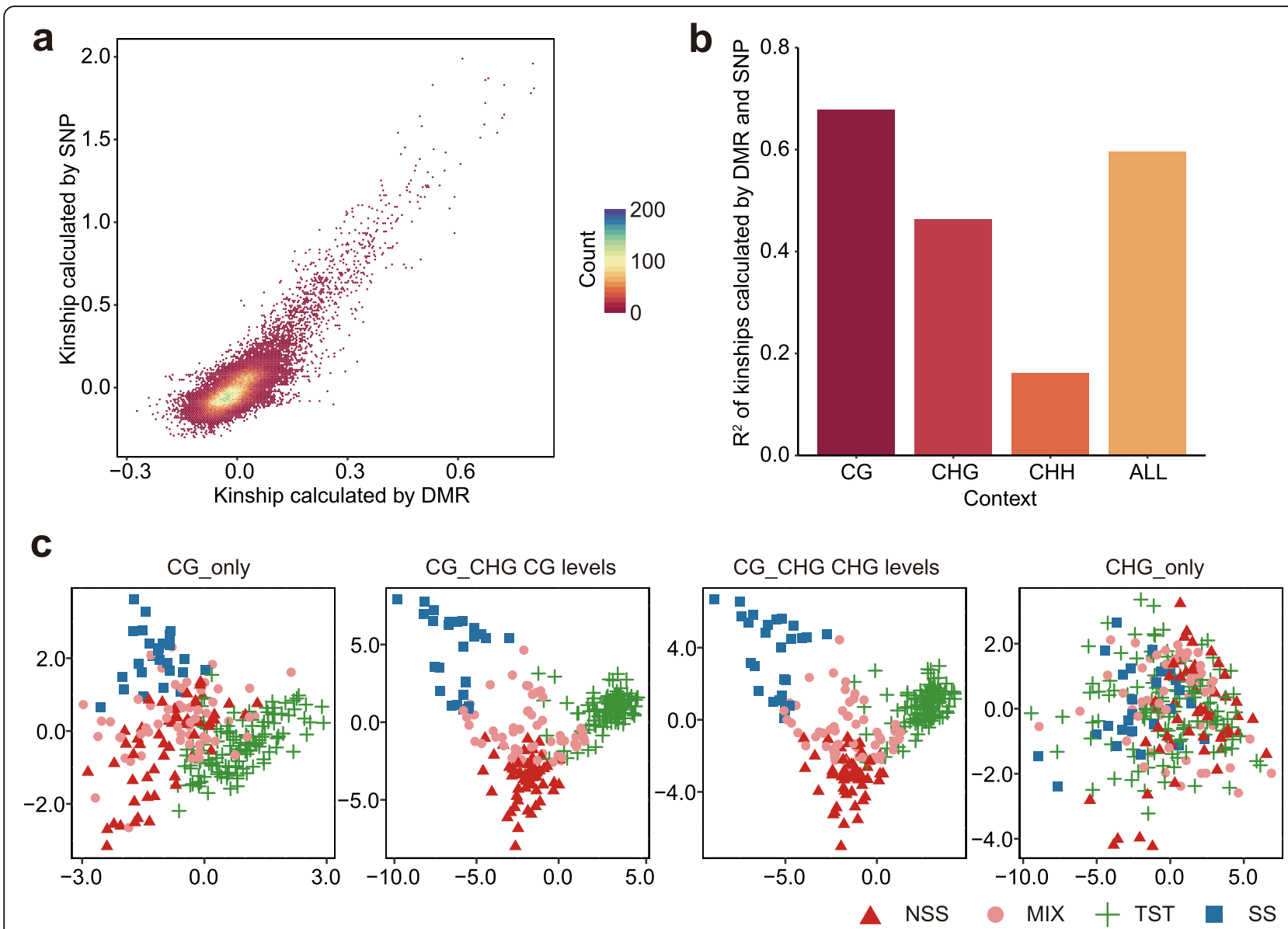

Fig. 2 Comparison of genetic distance calculated using either SNPs or DNA methylation. a The correlation of kinships between any two lines calculated by SNPs or DNA methylation in CG context. The color reflects the density of points. $\mathbf{b}$ The squared Pearson correlation coefficient $\left(R^{2}\right)$ between the two kinships calculated using either SNPs or the indicated DNA methylation. ALL means that the kinship was calculated using all three contexts. c PCA plots of context-specific DMRs. The color and shape of symbols reflect different subgroups of maize that are determined by SNPs. SS, stiff stalk; NSS, non-stiff stalk; TST, tropical or semi-tropical

nearby genetic changes. To investigate to what extent DMRs are tagged by SNPs and whether there are differences among different types of DMRs, we performed genome-wide association analysis (GWAS) with $\sim 1$ million high-quality SNPs using a mixed linear model that controls for both population structure and individual relatedness. We identified a total of 4336, 4096, and 1426 associations for CG, CHG, and CHH DMRs (Fig. 3a and Additional file 2: Table S2) respectively at a genomewide significance level of $5.15 \times 10^{-8}$ (Bonferroni-corrected $P$ value of multiple testing). Of the DMRs with associations, most shows significant association with only one SNP (Fig. 3b). Similarly, most of the SNPs associate with only one DMR (Fig. 3c). A close examination of the distance between the DMR and the associated SNPs shows that the distance is usually within $10 \mathrm{Mb}$ of each other (Fig. 3d). Thus, the SNPs were defined as local if the associated DMR is within $10 \mathrm{Mb}$ and were defined as distal if on a different chromosome. In agreement with the observation that no maize genotypes had global changes in DNA methylation in the association panel, no strong distal hotspots were identified (Fig. 3a). This suggests that the major methylation machinery was not compromised in the panel of inbreds used for this study.

Of the three sequence contexts, CG is most likely associated with SNPs either by local or by distal, and rarely by both (Fig. 3e). CHH DMRs are the least to show associations with SNPs. Importantly, $>60 \%$ of the DMRs do not have a significant association with any SNPs for each of the three sequence contexts (Fig. 3e), suggesting that these DMRs may represent unique information that is not captured by SNPs and therefore would not be represented in typical GWAS analyses. In some cases, the DMRs could arise from structural variants that may not be effectively tagged by SNPs. The set of structural variants identified by Yang et al. [34] were used for an association analysis with the DMRs (Additional file 1: Figure S5). This 


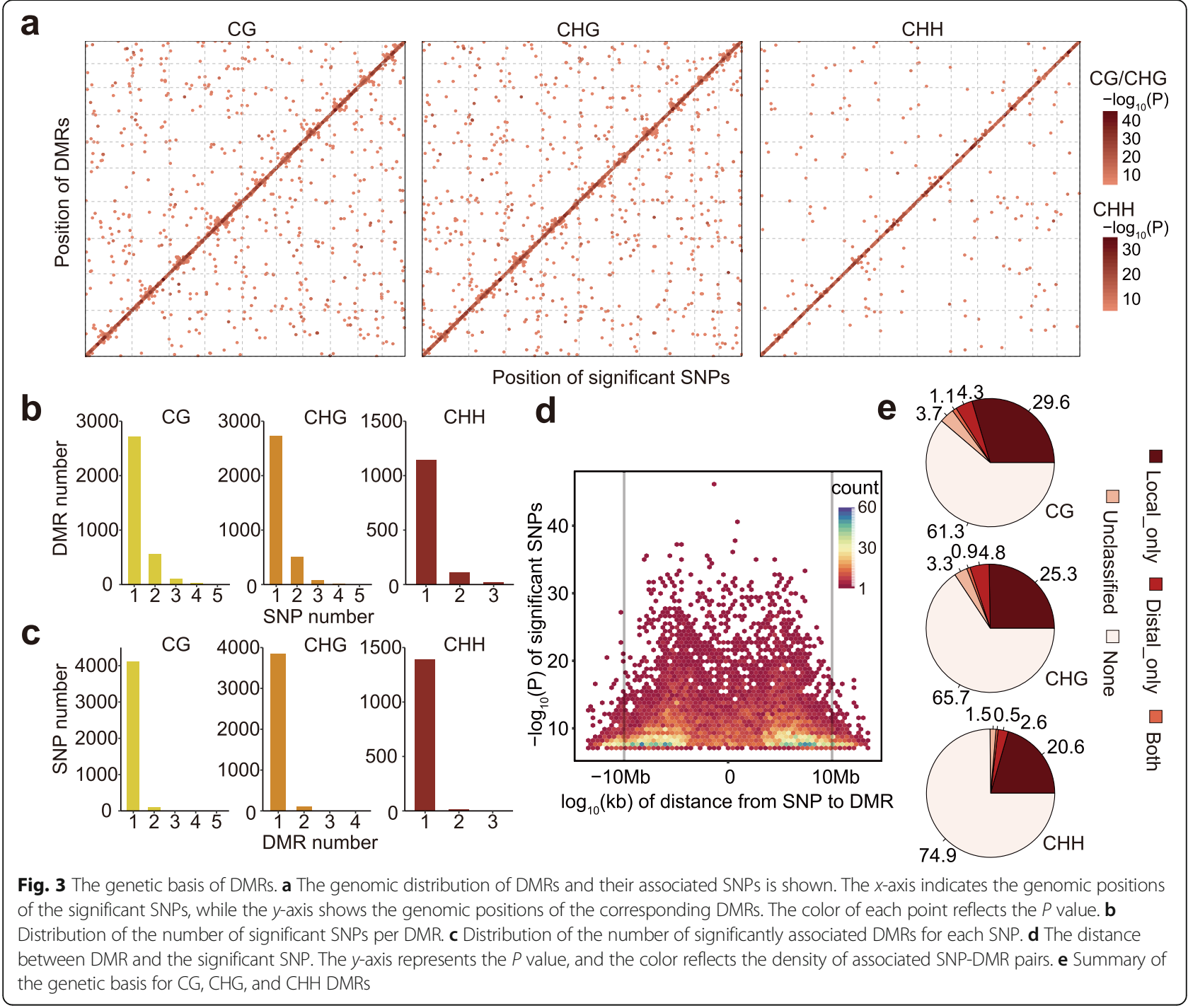

identified an additional $0.3-1 \%$ of the DMRs that do not have significant associations with SNPs that can be associated with a structural variant. Overall, these analyses suggest that a substantial portion of DMRs are not effectively captured by SNPs or structural variants.

\section{Variation in chromatin and genetic control of context- specific DMRs}

There is variation for the frequency of DMRs for different sequence contexts that exhibit significant association with SNPs. The proportion of DMRs with significantly associated SNPs is lower for CG_only (45\%) and CHG_ only (26\%) DMRs than that for CG_CHG DMRs (51\%) (Fig. 4a). This suggests that CG_only and CHG_only DMRs are less stable than CG_CHG DMRs, consistent with the above observation that CG_CHG DMRs have better power in differentiating maize subgroups. To investigate the properties of context-specific DMRs, we looked at the methylation levels of the other contexts in CG_only and CHG_only DMRs. For most of the CG_ only DMRs, the CHG levels are uniformly low across all genotypes (Fig. $4 \mathrm{~b}$ ), suggesting that these regions are not targets of Zmet2/Zmet5, which maintains CHG methylation. In contrast, most of the CHG_only DMRs have high levels of CG methylation in all genotypes (Fig. 4b), suggesting that these regions are consistent targets of CG methylation maintaining enzyme, MET1. There is also variation in the chromatin features of contextspecific DMRs (Fig. 4c, d). CG_only DMRs tend to have low levels of both H3K9me2 and H3K27me3 marks. This suggests that CG_only DMRs are less likely to occur in regions with repressive chromatin environments, in agreement with the findings that they are enriched in genic regions and likely represent differential gene body methylation (Additional file 1: Figure S2). CHG_only DMRs tend to have higher H3K9me2. The 


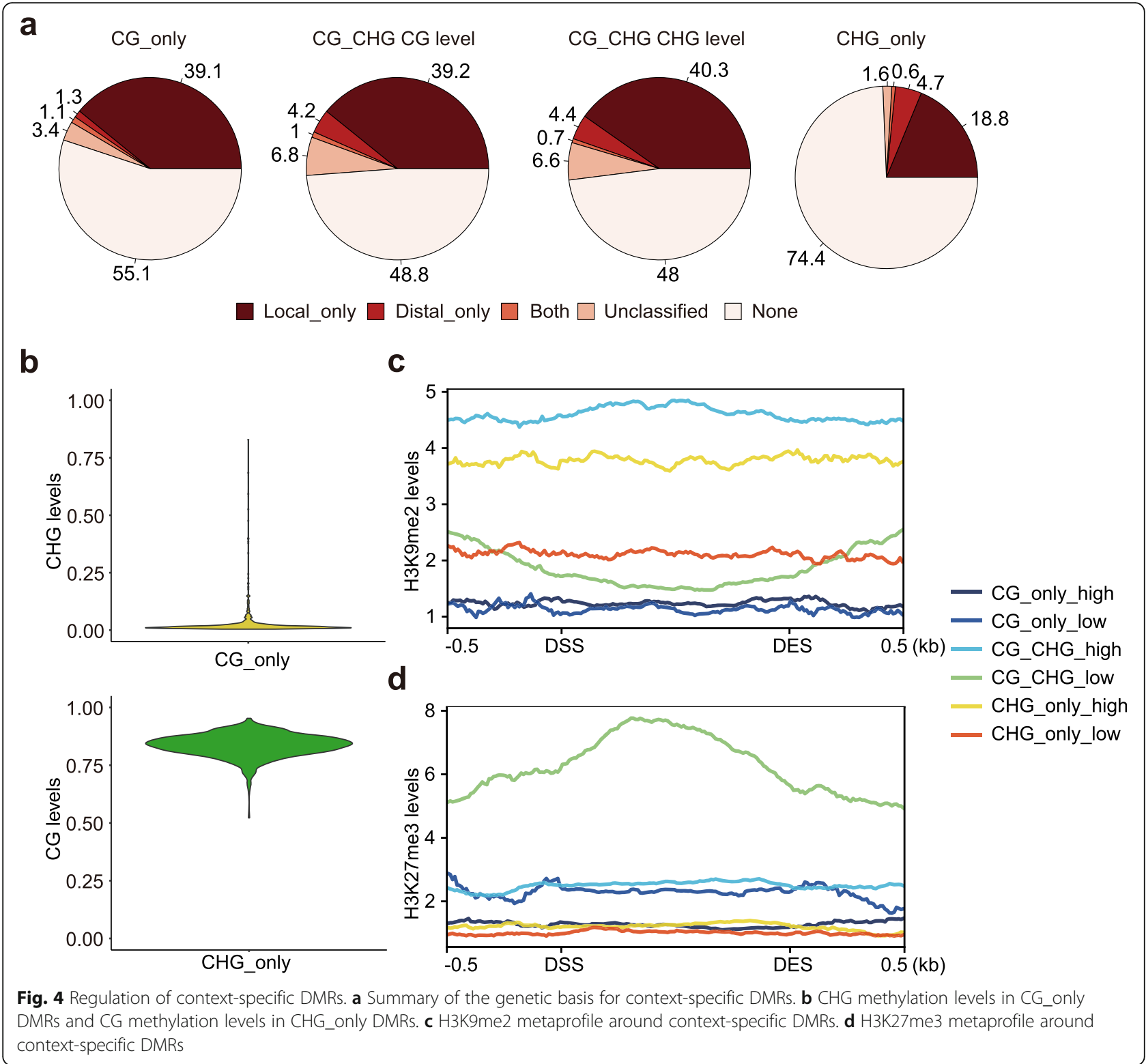

high levels of $\mathrm{H} 3 \mathrm{~K} 9 \mathrm{me} 2$ were only observed in the subset of CHG_only DMRs that have high DNA methylation in the B73 where H3K9me2 data was collected, not in the DMRs where DNA methylation is low in B73. This agrees with the fact that CHG methylation and H3K9me2 form a self-reinforcing loop [35]. In contrast to CG_only and CHG_only DMRs, CG_CHG DMRs have high levels of both H3K9me2 and H3K27me3. Interestingly, the high levels of $\mathrm{H} 3 \mathrm{~K} 9 \mathrm{me} 2$ occur in DMRs where DNA methylation is high in B73 (the genotype where $\mathrm{H} 3 \mathrm{~K} 9 \mathrm{me} 2$ and $\mathrm{H} 3 \mathrm{~K} 27 \mathrm{me} 3$ were collected), while the high levels of H3K27me3 occur in DMRs where DNA methylation is low in B73 (Fig. 4c, d). This agrees with the reports that DNA methylation and H3K27me3 are generally repulsive [36].
DMRs are associated with natural variation in gene expression

To investigate the association between DNA methylation and gene expression, we performed GWAS using gene expression as the dependent variable and DNA methylation as the independent variable. The expression data was from kernels 15 days after pollination. At $P<1 \times 10^{-6}$ (Bonferroni's correction for multiple test, $0.01 / N, N$ is the number of DMRs), 1389 significant associations between expression and methylation levels were detected, with CG, CHG, and $\mathrm{CHH}$ DMRs having 538, 562, and 289 associations, respectively (Fig. 5a, Additional file 2: Table S3). The majority of the significantly associated DMRs were located on the same chromosome as the genes, and the distance between the DMR and the gene is usually less than $1 \mathrm{Mb}$ 

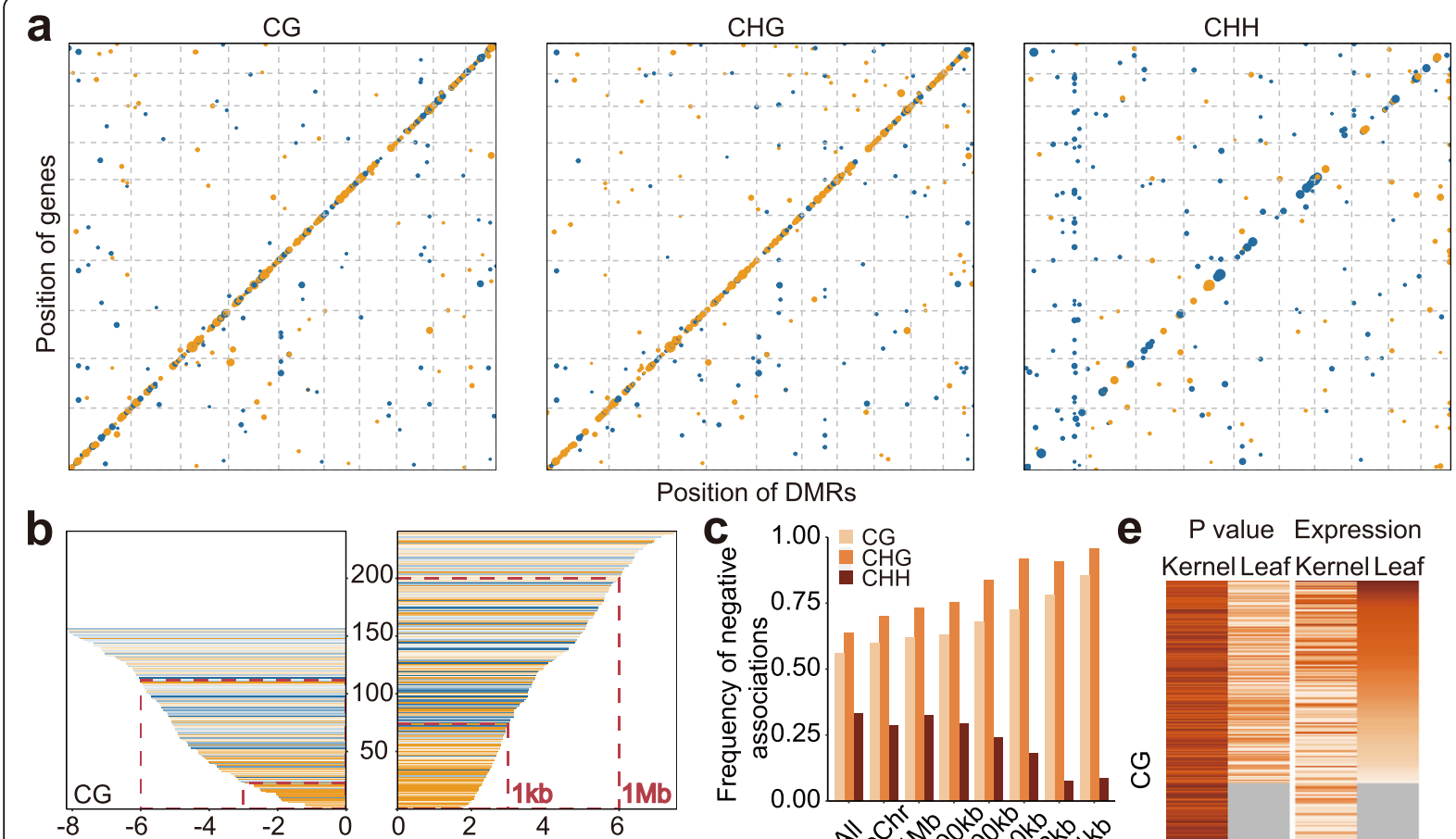

Position of DMRs
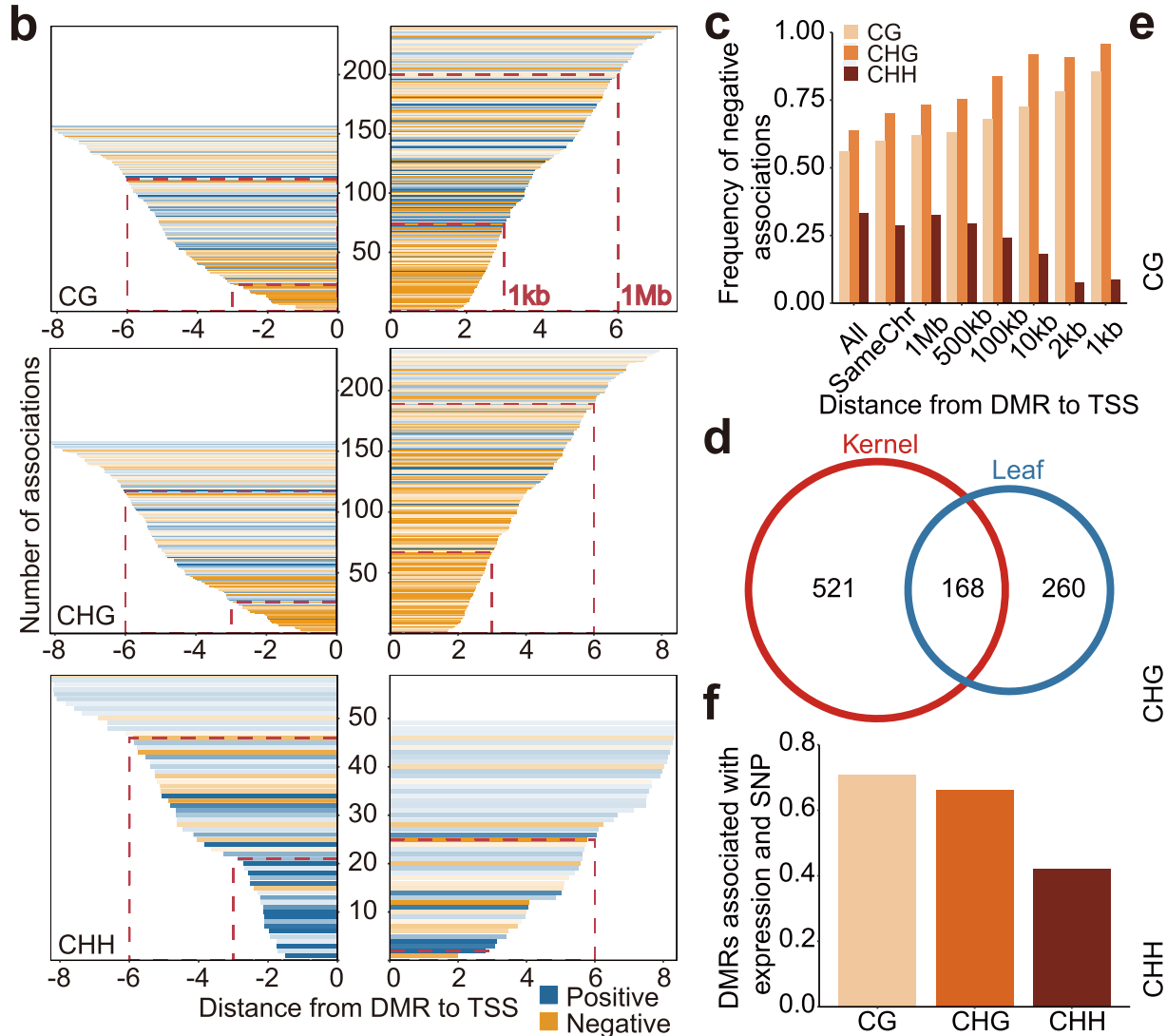

P value Expression Kernel Leaf Kernel Leaf
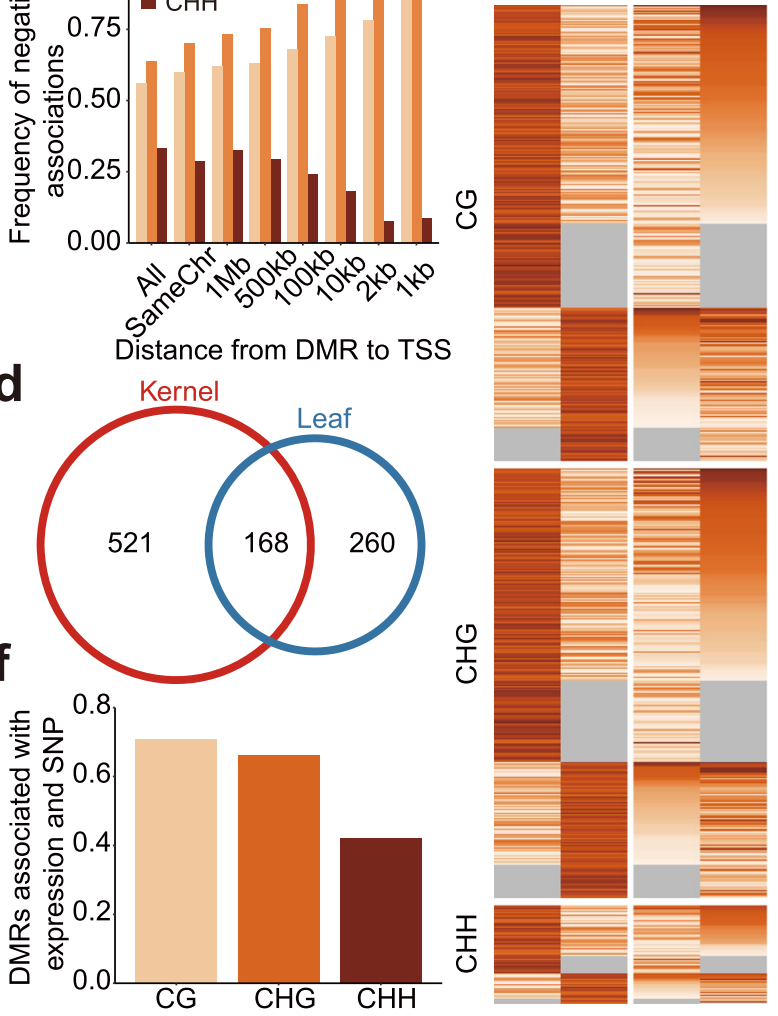

$\stackrel{0}{\mathrm{~T}}$

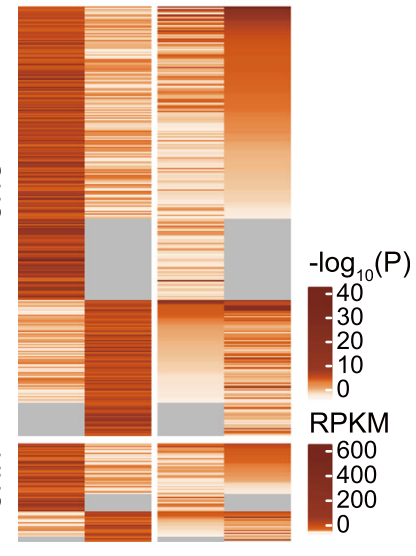

Fig. 5 Associations between DMRs and gene expression. a The genomic distribution of genes and their associated DMRs. The $x$-axis indicates the genomic positions of DMRs, while the $y$-axis is the genomic positions of corresponding genes. The color reflects the direction of association between gene expression and DMRs, with blue for positive associations and orange for negative associations. Note there are more orange dots (negative associations) in the plots of CG and CHG methylation, and more blue dots (positive associations) in the plot of CHH methylation. b The distance between the DMR and the gene transcription start site (TSS) is evaluated for DMR-gene expression associations. The $x$-axis is the log $\log _{10}$ (downstream) or - $\log _{10}$ (upstream) value of the distance from DMR to the TSS, while the $y$-axis is the number of associations. The color indicates the direction of associations with darker shades representing lower $P$ value. c Summary plot for the proportion of negative associations for DMRs with varying distance to gene TSS. Note the closer the DMR to gene, the more negative associations for CG and CHG methylation and the more positive associations for CHH methylation. $\mathbf{d}$ Comparison between leaf and kernel expression data using associations within $1 \mathrm{Mb}$ from DMR to TSS. e The expression levels of genes in leaf (kernel) which only show significant associations in kernel (leaf) tissue. The left plot shows $P$ value of significant gene-DMR associations, and the right plot shows levels of gene expression. Gray color means that the data are not available because of low/no expression of the gene in that tissue. $\mathbf{f}$ The proportion of DMRs associated with gene expression that also have a significant association with SNPs 
(Additional file 1: Figure S6a). The majority of DMRs are associated with only one gene, arguing against the presence of distal hotspots (Fig. 5a).

There are more negative associations for CG and CHG DMRs (Fig. $5 \mathrm{a}-\mathrm{c}$ ). In contrast, $\mathrm{CHH}$ DMRs tend to be positively associated with gene expression. These trends become stronger the closer the DMR is to the transcription start site (TSS), especially for DMRs within $1 \mathrm{~kb}$ of TSS (Fig. 5b, c). Many of the negative associations for CG and CHG were located downstream of the TSS (Fig. 5b), suggesting that high CG and CHG methylation within the gene body are likely to associate with reduced expression. It is observed that all three sequence contexts, CG, CHG, and $\mathrm{CHH}$, from the same DMR can associate with expression of the same gene though the direction of association could be different (Additional file 1: Figure S6b). Interestingly, CG_CHG DMRs are $\sim 3$ times more likely to be associated with gene expression compared with CG_only and CHG_only DMRs (Additional file 1: Figure S6c). The CHG methylation from either CG_CHG DMRs or CHG_only DMRs tend to be negatively associated with gene expression, while CG methylation shows negative associations when it covaries with $\mathrm{CHG}$ methylation but shows positive associations (>75\% cases) when in CG_only DMRs (Additional file 1: Figure S6d). Thus, the association between gene expression and DNA methylation is dependent upon sequence context.

To assess how DMRs are associated with gene expression in different tissues, we explored the association between DMRs and gene expression in a second expression dataset derived from leaf tissue, and compared with the associations from the developing kernels. We identified a total of 1096 associations in the leaf tissue, compared to 1389 in the kernel tissue. There are 689 and 428 DMR-gene associations which are located within $1 \mathrm{Mb}$ of each other in kernel and leaf, respectively, including 168 associations that were identified in both tissues (Fig. 5d). The shared associations include 74, 79, and 15 for CG, CHG, and CHH DMRs respectively. While some DMRs show common associations, there are many DMRs that only associate with gene expression in one of the tissues (Fig. 5d). A close examination of the data revealed that $3.7-12.5 \%$ of tissuespecific associations are significant in another tissue if less stringent criteria were applied, and 17 29\% were due to tissue-specific expression patterns of genes (Fig. 5e). For the remaining gene-DMR associations, expressions of the genes were detected in both tissues but significant associations were detected only in one tissue. This suggests the full set of genes with expression levels influenced by DNA methylation variation can only be documented through the analysis of many different tissues or growth conditions.
We assessed whether the DMRs that were significantly associated with gene expression were enriched for associations with SNPs. A subset (42-71\%) of the DMRs that are associated with gene expression also have significant association with SNPs, with higher proportions for CG and CHG DMRs than for CHH DMRs (Fig. 5f). The remaining $29-58 \%$ of the DMRs associated with gene expression are not significantly associated with SNPs. Similar patterns were found for context-specific DMRs (Additional file 1: Figure S6e). Together, these results suggest that DMRs, whether or not tagged by SNPs, can explain variation in gene expression.

\section{A causal relationship between DMRs and variation in gene expression}

Differential DNA methylation could be a cause or a consequence of differential gene expression. In an attempt to address this question, we compared two models using the Mendelian randomization test (Additional file 1: Figure S7a). The first model assumes a DMR is a cause of differential gene expression. We selected instrumental SNPs that show strong association with DMRs but not with gene expression, so that the effect of the SNP on gene expression was because of the combined effect of SNP on DMR and the effect of DMR on gene, which is called the predicted effect. We then compared this predicted effect with the observed effect. Interestingly, there is a strong correlation between the predicted effect and the observed effect. In contrast, the correlation is much smaller in a second model where DMR is a consequence of differential gene expression. The difference in correlation between the two models is observed in both the kernel and leaf tissues (Additional file 1: Figures S7 and S8). It is also observed for each of the three sequence contexts irrespective of the direction of association and the distance between DMR and gene (Additional file 1: Figures S7 and S8). These support an idea that DMR is more likely to be a cause of differential gene expression.

Next, we set to validate the causative role of DNA methylation on gene expression by using methylome mutants. We took advantage of the RNA-seq data from kernel tissue of the $d d m 1$ double mutant which disturbs DNA methylome dramatically [37]. We assessed whether the genes detected as exhibiting natural variation for DNA methylation linked to DMRs in our population would also show changes in expression in the $d d m 1 \mathrm{mu}-$ tant. As the $d d m 1$ mutant has limited changes in CG and there is no available mutant in maize that disturbs CG methylation, we focused on CHG and CHH methylation. For CHG DMRs, 67\% of DMR-gene associations could be supported in $d d m 1$ mutant with the direction of association being the same in the natural population and in the $d d m 1$ mutant, compared to $\sim 50 \%$ for random control which uses the top 5000 non-significant 
DMR-gene associations (Additional file 1: Figure S7b). For $\mathrm{CHH}$ DMRs, $62 \%$ were supported. Interestingly, both negative and positive associations from natural variation could be supported by the changes in expression in the $d d m 1$ mutant. These results provide further support that DNA methylation can have both a negative and a positive causal effect on gene expression.

\section{Natural DMRs explain phenotypic diversity}

If DMRs are tightly linked to nearby SNPs, then the potential phenotypic impact of this heritable information would be captured in SNP-based GWAS scans. However, if DMRs are not tightly linked to SNPs, then the variation in DNA methylation will reflect novel information not included in SNP-based scans. To identify whether DMRs can affect phenotypic variations both dependent upon and independent of SNPs, we performed GWAS using DMRs as independent variables and phenotypic data as dependent variables. We used the metabolic data that has been generated for this diverse panel in a previous study [38]. A total of 986 unique metabolic traits were measured in 3 independent environments. At a genome-wide significance level of $0.05 / N$ ( $N$ is the number of DMRs which is 8864, 9759, and 5075 for CG, CHG, and CHH DMRs, respectively), 156 traits $(15.8 \%)$ show significant associations with a DMR in at least 1 environment (Fig. 6a, Additional file 2:
Table S4). The majority of the traits (76\%) show associations with one DMR (Fig. 6a). There are 12 traits that are associated with the same DMR in at least 2 environments, suggesting that the effect of some DMRs is stable and reproducible. In total, $43 \mathrm{CG}, 45 \mathrm{CHG}$, and $63 \mathrm{CHH}$ DMRs were found to be significantly associated with metabolic traits (Fig. 6b). Some DMRs can associate with more than 1 metabolic trait, leading to a total of 250 DMR-trait associations. Interestingly, many of the significantly associated DMRs are not significantly associated with SNPs, with higher proportions for $\mathrm{CHH}$ DMRs than for CG and CHG DMRs (Fig. 6c). There are many traits that only show associations with DMRs but not with SNPs (Additional file 2: Table S4), suggesting that DMRs may be more predictable for phenotypic variation than SNPs for some traits.

One example is shown in Fig. 6d, e to demonstrate the effect of a DMR on phenotype. Chrysoeriol di-C-hexoside (chr di-C) is one type of flavonoid where chrysoeriol is linked to two sugar groups by an $\mathrm{O}$-glycosidic bond. The flavone chrysoeriol is a derivative of chalcone (Fig. 6d). A total of eight significant associations with DMRs were identified for chr di-C content (Fig. 6e, Additional file 2: Table S4). The most significant associations were on chromosome 1 around $48.4 \mathrm{Mb}$. All three sequence contexts were found to be significant in two environments (Additional file 1: Figure S9a, Additional file 2: Table S4).

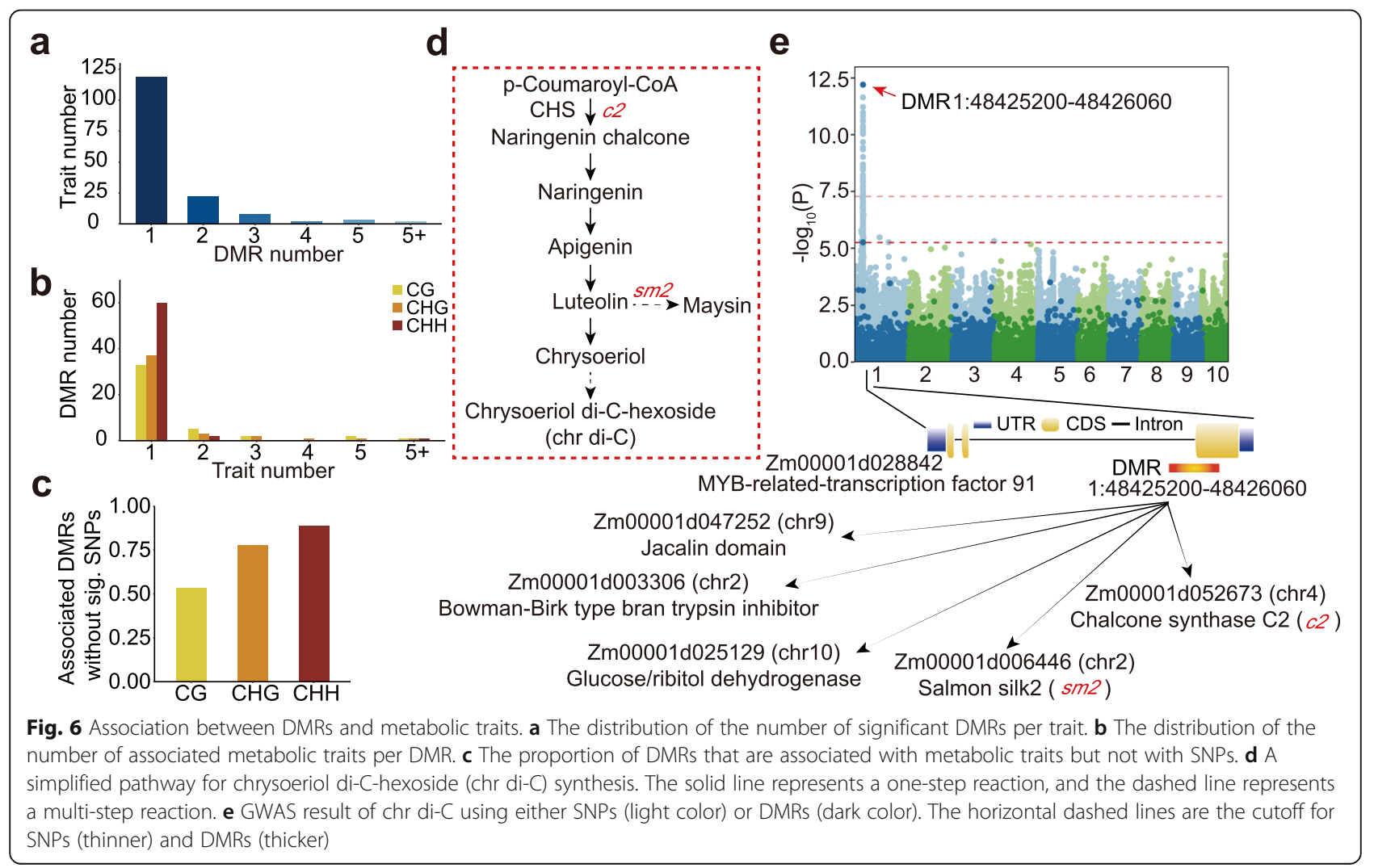


The DMRs were located within a gene annotated as a MYB transcription factor which can bind promoters of flavonoid synthesis-related genes (Fig. 6e, Additional file 1: Figure S9b). This DMR was also found to associate significantly with the expression of five genes. Three of these five genes were annotated to have a role in flavonoid metabolism, including one glucose/ribitol dehydrogenase, one chalcone synthase (CHS), and salmon silk 2 (sm2). $\mathrm{CHS}$ is the major enzyme responsible for chalcone (Fig. 6d), which is the precursor for chrysoeriol. The sm2 gene is also involved in the biosynthesis pathway of chrysoeriol (Fig. 6d). These five genes are located on different chromosomes from the DMR. It is possible that the transcription factor where the DMR is located can regulate the expression of these genes, leading to variations in chr di-C. This is supported by the fact that the expression of the five genes was associated with chr di-C content (Additional file 1: Figure S9c). The effect of this DMR may be related to genetic variation as many SNPs that are significantly associated with this DMR have been identified (Additional file 1: Figure S9d). These SNPs were located $\sim 100 \mathrm{~kb}$ downstream from the DMR, and many of the SNPs were also significantly associated with chr di-C (Additional file 1: Figure S9b). However, the association for the DMR is the highest in one of the environments (Fig. 6e). These results suggest an important role of DNA methylation in contributing to phenotypic diversity.

\section{Discussion}

\section{The genetic architecture of DMRs}

In this study, we profiled DNA methylation across a diverse panel of 263 maize inbred lines. Extensive variation was found in all three sequence contexts across the ten maize chromosomes. One interesting discovery is that there is no obvious natural mutant with compromised DNA methylation in this panel that includes abundant genetic diversity. All 263 diverse inbreds have similar levels of DNA methylation, showing either high or low methylation at specific loci. The lack of distal hotspots that are associated with methylation machinery provides further support of the lack of natural variation for DNA methylation machinery. This is in contrast to studies in Arabidopsis. For example, the VIM gene, which controls for CG methylation, was identified in a natural Arabidopsis accession that shows genome-wide loss of DNA methylation [33]. The CMT2 gene, which controls $\mathrm{CHH}$ methylation in heterochromatic regions, also has loss-offunction genetic variations in natural accessions [17, 32]. The lack of natural mutant of DNA methylation in maize suggests an important role of DNA methylation in plants with large genomes. This is well supported from studies in rice and maize, which show that genome-wide loss of DNA methylation leads to severe phenotypic effects and seed lethality in many cases [3-6, 39, 40].
Similar to prior results in other plant species [12, 16-18], a subset of regions with variable methylation can be associated with SNPs. This is true for all sequence contexts with variability in the proportion of DMRs for different sequence contexts that can be linked to SNPs. However, a large proportion $(>60 \%)$ of DMRs are not tagged by SNPs, suggesting that there is unique information in the DNA methylome. This was further supported by the observation that DNA methylation that is not associated with SNPs can effectively differentiate maize subgroups (Additional file 1: Figure S3b). These results support the concept that DMR can carry unique heritable information independent of genetic variation and that this information would not be captured in current SNP-based GWAS approaches. We should note, however, lack of association with SNPs does not necessarily mean that these DMRs are purely epigenetic. Low frequency SNPs which by design are not considered in GWAS could be associated with DMRs. Similarly, structural variants, which show low LD with SNPs, could also be associated with DMRs (Additional file 1: Figure S5a). We observed that DMRs with high MEF are more likely to associate with genetic variations, but we do find DMRs with high MEF that do not associate with genetic variations even after we consider the structural variants (Additional file 1: Figure S5b). These non-tagged DMRs with a relatively high MEF should be good candidates for pure epigenetic variants.

It is worthwhile to note that the capture-based method to assay DNA methylation levels in this study will have some limitations in monitoring genome-wide rates or variability and detecting the full set of information contained in the methylome. The capture-based approach focuses on a limited number of regions throughout the maize genome. These regions were chosen based on our previous knowledge of DNA methylation in maize genome $[13,30]$. We purposely chose many regions with variation in DNA methylation that can be uniquely mapped across a very diverse set of lines. These regions represent portions of the genome that are more conserved across different maize lines. Considering the limited portion of the genome represented by our capture probes $(15 \mathrm{Mb}$, or $0.6 \%$ of the entire genome), it is very likely that there are more regions showing variation in DNA methylation, and pure epigenetic information with important biological significance may widely occur in maize.

\section{DNA methylation contributes to differential gene expression}

Both positive and negative associations between DNA methylation and gene expression have been proposed $[13,23,30,41]$. We found that this relationship depends heavily on sequence contexts and the position of DMRs relative to gene TSS. DMRs located within $1 \mathrm{~kb}$ of the TSS tend to be enriched for negative associations for CG 
and CHG methylation and positive associations for $\mathrm{CHH}$ methylation. Another interesting question is whether differential methylation is a cause or consequence of differential gene expression. Previous studies have shown that loss/gain of DNA methylation in methylome mutants is usually accompanied by changes in gene expression [16, 42-44]. This suggests that changes in DNA methylation can be a cause of differences in gene expression. A genome-wide assay of natural variation of DNA methylation on gene expression in model plant Arabidopsis also suggests that DNA methylation is more likely the cause rather than the consequence of variation in gene expression [23]. However, there are also examples where DNA methylation is a consequence of differential gene expression [45]. Our genome-wide analysis favors the possibility that some DMRs are causes of variation in gene expression. The negative effect of DNA methylation on gene expression is likely due to a repressive chromatin environment associated with DNA methylation, while the positive effect of DNA methylation is likely attribute to the recruitment by DNA methylation of specific transcription factors to enhance gene expression [46].

\section{Epigenetic information contributes to phenotypic diversity}

Many agronomic traits have a complex genetic basis with numerous minor effect QTLs [47-49]. The fact that only a portion of the phenotypic variance of these traits can be explained by SNPs has led to the suggestion that other types of heritable information may be involved. The growing use of SNP information for performing genomic selection in plant breeding has increased our reliance upon SNPs to explain phenotypic variation. We were interested in exploring the potential for DNA methylation to provide information about gene expression or phenotypic differences that may not be present in SNP profiles. There are examples for which DNA methylation, rather than genetic variation, at specific loci is the cause of a phenotypic change [50]. It was reported that DNA methylation can lead to phenotypic diversity independently of genetic variation based on QTL mapping using epi-recombinant inbred lines that share same genetic background but show variations in DNA methylation patterns $[51,52]$. Here, we found that many of the DMRs were not captured by SNPs, some of which also show significant associations with gene expression and phenotypic diversity. Our results suggest that DNA methylation can affect phenotypic diversity under at least two circumstances. First, DNA methylation is a bridge between genetic variation and phenotype. In this scenario, a genetic difference (SNP or structural variant) is the cause of phenotypic variations and methylation changes are trigged by the genetic variation. Second, pure epigenetic information is contained within DMRs that are independent of genetic variation. There are many metabolic traits for which significant associations were only identified for DMRs but not for SNPs. Our study highlights the potential for DNA methylation variation that is not identifiable through SNPs and for this variation to influence gene expression and traits.

\section{Conclusions}

In conclusion, DNA methylation was investigated at a population level in maize. Abundant variation in DNA methylation was identified, only some of which is effectively captured by SNP associations. Variations in DNA methylation can separate maize subgroups, associate with differential gene expression, and contribute to phenotypic diversity. This study represents the first effort to perform genome-wide association analysis using epigenetic data (DNA methylation) in a crop species. The findings that DMRs not tagged by genetic variation are prevalent and can cause phenotypic variation suggest that DNA methylation is a candidate to explain a portion of the heritability that is not effectively captured by SNPs.

\section{Methods}

Materials

The maize inbred lines used in this study are from a global collection and represent a wide range of diversity [53]. These lines were grown using standard greenhouse conditions until V3, and the third leaf was collected and frozen in liquid nitrogen. Genomic DNA was extracted using the standard cetyl-trimethyl-ammonium bromide method. Two approaches were used to verify material authenticity. First, five polymorphic InDel markers were used to genotype the entire panel, and the results were compared with that from previous genotyping. Only lines with consistent genotyping were kept. Second, SNPs were de novo called based on the sequencing data generated in this study using BS-SNPer [54] and were compared with the SNP data from a previous study [55]. The genotypes with consistency of more than $90 \%$ were kept, resulting in a total of 263 lines.

\section{Library preparation, sequencing, and mapping}

The design of probes for the capture regions can be found in a previous study [29]. Briefly, $15.7 \mathrm{Mb}$ of the maize genome was used for probe design based on B73 reference genome (AGPv2). These regions include all the regions from our first version of capture probes [28], as well as additional regions that are selected based on our study of DNA methylation in maize. These additional regions mainly include DMRs between genotypes (B73 and Mo17 or Oh43), DMRs among five tissues (seedling leaf, mature leaf, shoot apical meristem, anther, and immature ear), DMRs identified during tissue 
culture, promoters of potential hidden genes which are defined as genes that have high DNA methylation in B73 genome and low/no expression in various B73 tissues, and $\mathrm{mCHH}$ island. Information of the capture regions can be found on Data Repository for University of Minnesota [56].

Bisulfite-converted sequencing libraries were constructed using a previously published method for capturing specific regions [28]. The libraries were sequenced on HiSeq 2500 platform using 125 cycles and paired-end mode. After trimming adapters by Trim Galore [57], reads were mapped to the $\mathrm{B} 73$ reference genome version 4 [31] using BSMAP allowing up to five total mismatches [58]. Reads that are mapped uniquely were kept. Duplicate reads and reads that are not properly paired were also removed using Picard Tools [59] and BamTools [60]. The methylation levels at each individual cytosine were called using methratio.py in BSMAP.

\section{DMR identification}

The DMRs among all the genotypes were identified using a two-step method. In step 1, DMRs were identified between two lines. The maize genome was divided into non-overlapping $20 \mathrm{bp}$ windows, and the methylation levels of each $20 \mathrm{bp}$ window that have cytosine sites with at least $2 \times$ coverage were calculated for each line. Sixteen lines with high read coverage and genetic diversity were selected as group I, with all the other lines as group II. Each line from the first group was then compared with each of the line from the second group. For each pair-wise comparison, the 20-bp windows with CG and CHG difference of greater than $60 \%$ were kept. For $\mathrm{CHH}$, the windows that meet the following criteria were kept: over than $20 \%$ difference between the contrasted genotype, having one genotype of less than 5\% methylation, and the other genotype of greater than $25 \%$ methylation. The 20-bp windows that are within $50 \mathrm{bp}$ of each other and have the same direction were then merged for each comparison, and the merged regions with at least three continuous 20-bp windows with data were classified as DMRs.

In step 2, the DMRs from each pair-wise comparison were merged using the following pipelines. First, DMRs within $200 \mathrm{bp}$ of each other were merged using BEDTools [61]. Second, the merged DMRs were divided into $20 \mathrm{bp}$ windows and the percentage of contrasting pairs with significant difference in DNA methylation for each $20 \mathrm{bp}$ window was calculated. This percentage was then normalized to the window with the highest percentage. The window with the normalized value of less than 0.4 was dropped, and the DMRs with at least three continuously eligible windows were kept. Third, each remaining DMR was required to meet the following criteria in $>$ $60 \%$ of the lines: $\geq 2 \times$ coverage, $\geq 6$ cytosine sites, and $\geq$
$2 / 3$ of the cytosines within the region were covered. The above procedure was done separately for the three sequence contexts. Lastly, regions were classified as CG or CHG DMRs if the difference in methylation was greater than $60 \%$ between the second top highest and lowest lines for CG or CHG contexts, and was called as $\mathrm{CHH}$ DMR if the second highest line having a $\mathrm{CHH}$ level of > $25 \%$ and the second lowest line having a $\mathrm{CHH}$ level of $<$ $5 \%$. This would eliminate DMRs that are unique to a single line.

To define context-specific DMRs, the following criteria were used. For CG DMRs, if CHG methylation difference was greater than $60 \%$ and the squared Pearson correlation coefficient $\left(R^{2}\right)$ between CG and CHG levels were more than 0.8 , the CG DMR was called as CG CHG DMRs. If CHG methylation difference was less than $20 \%$ and $R^{2}$ was less than 0.2 , the CG DMR was called as CG_only DMR. Similarly, for CHG DMR, if CG methylation difference was greater than $60 \%$ and the $R^{2}$ between CG and CHG levels were more than 0.8, the CHG DMR was called as CG_CHG DMRs. If CG methylation difference was less than $20 \%$ and $R^{2}$ was less than 0.2 , the CHG DMR was called as CHG_only DMR.

\section{Comparison of relatedness using SNPs or DNA methylation}

Two methods were used to compare the individual relatedness generated by SNP and DNA methylation levels, respectively. First, the $\mathrm{R}$ package, pcaMethods, was used to perform PCA analysis [62]. The algorithm was ppca, and the number of components was set as 3 . Second, GCTA was used to calculate individual relatedness based on SNPs and OSCA was used to generate individual relatedness based on DNA methylation data $[63,64]$. The $R^{2}$ of the two matrices with the diagonal value being removed was used to quantify the relationship between SNP and DNA methylation. The data for the three DNA methylation sequence contexts were calculated either separately or together.

\section{Identification of subgroup-specific DMRs}

A one-way ANOVA was conducted to compare DNA methylation levels among three maize subgroups: SS, NSS, and TST. The significant DMRs at the $P<0.001$ level were selected for post hoc comparisons using the Tukey HSD test. The DMRs that are significant at the $P<0.001$ level among any two subgroups were defined as subgroup-specific DMRs.

\section{MEF calculation}

For CG and CHG DMRs, if the difference in methylation was greater than $60 \%$ between any two lines, the line with higher methylation was assigned into "High" group and the other line was assigned into "Low" group. DMRs for which $>50 \%$ of the lines could be assigned to either 
"High" or "Low" group were kept. The MEF was calculated by the number of lines in the group with fewer lines divided by the sum of lines in the two groups. For $\mathrm{CHH}$ DMRs, the methylation difference was that the higher line having a $\mathrm{CHH}$ level of $>25 \%$ and the lower line having a $\mathrm{CHH}$ level of $<5 \%$. The rest is similar as CG and CHG DMRs.

\section{Methylation QTL mapping}

An integrated map of 1.25 million SNPs from a previous study [55] was converted to B73 version 4 coordinates using alignment of the sequences around the SNPs. The SNPs were retained if the position of the B73 V4 reference genome has exactly the same nucleotide as the B73 SNP from the conversion based on alignment. The SNPs were further filtered to retain those that have a minor allele frequency (MAF) of greater than 0.05 in the lines with methylation data. For each DMR, the methylation value was normalized using rank-based inverse normal transformation. A mixed linear model was used to perform GWAS by controlling for population structure and family relatedness [65]. The kinship was calculated by EMMAX, and the population structure was estimated by ADMIXTURE [66]. A Bonferroni-corrected $P<5.15 \times$ $10^{-8}(0.05 / N, N=971,267)$ was used to determine significant associations. The association between structural variants and DMRs was calculated by the same way with a Bonferroni-corrected $P<1.84 \times 10^{-5}(0.05 / N, N=$ 2711) for structural variants identified based on comparison between $\mathrm{B} 73$ and SK and $P<2.01 \times 10^{-5}$ for structural variants identified based on comparison between Mo17 and SK $(0.05 / N, N=2484)$. As some associations may be caused by SNPs in LD, two criteria were used to filter these associations. First, LD analysis was performed using Haploview for all significant SNPs and only independent SNPs $\left(r^{2}<0.1\right)$ were kept [67]. The remaining SNPs should not be in LD with any other SNPs that are associated with the same DMR. Second, the median values of methylation for each haplotype was calculated and only associations having methylation difference greater than 0.05 were remained. According to the distance between DMRs and the associated SNPs, SNPs within $10 \mathrm{Mb}$ of DMRs were defined as local SNPs and SNPs that are on another chromosome were distal SNPs. The SNPs that are on the same chromosome of DMRs but $>10 \mathrm{Mb}$ away were defined as unclassified SNPs. DMRs that only had local SNPs were defined as "Local_only" type. DMRs with only distal associations were defined as "Distal_only." DMRs that have both local and distal SNPs were defined as "Both." DMRs that have unclassified SNPs were defined as "Unclassified." DMRs that do not have associated SNPs were defined as "None."

\section{Chromatin features of context-specific DMRs}

The ChIP-seq data of histone modifications, H3K9me2 (SRR1482372) [68] and H3K27me3 (SRR5436222) [69], were downloaded from NCBI. The sequencing reads were processed using Trim Galore to remove adapters and nucleotides with bad quality. Reads that passed quality control were mapped to the reference genome of B73 version 4 using Bowtie2 [70] with default parameters. The metaplots showing read coverage around context-specific DMRs were generated using deepTools [71].

\section{Association between DMRs and quantitative traits including gene expression and metabolic traits}

A single-marker test based on mixed linear model was used to analyze the association between DMR and gene expression/metabolic traits. Metabolic traits were from the study of Wen et al. [38]. There are 192 lines that have both metabolic data and methylation data. Two RNA-seq datasets were utilized, one with RNA-seq data for kernels 15 days after pollination [72] and another with RNA-seq data for leaf tissue. For kernel tissue, there are 193 lines that have both expression and methylation data. For leaf tissue, there are 108 lines with expression and methylation data. The RNA-seq reads were re-mapped to B73 v4 genome using TopHat2 [73] with the default parameters. Only the genes that have expression and methylation data in at least $60 \%$ of the lines were used. A two-step residual model was applied. In the first step, gene expression or metabolic traits $(Y)$ were normalized using rank-based inverse normal transformation method. The normalized value was then fit in the following model to get a residual expression level $\left(Y^{\prime}\right)$ after controlling for the effect of population structure $(Q)$ and individual relatedness $(K)$.

$$
Y=Q+K+\varepsilon 1
$$

In step 2, the newly calculated $Y^{\prime}$ was then used as the dependent variable to fit a linear regression model with DNA methylation $(X)$ as the independent variable.

$$
Y^{\prime}=\beta X+\varepsilon 2
$$

The resulting $P$ value was corrected using the Bonferroni method $(P<0.01 / n)$, and the value was $1.13 \times 10^{-6}$ for CG, $1.02 \times 10^{-6}$ for $\mathrm{CHG}$, and $1.97 \times 10^{-6}$ for $\mathrm{CHH}$. The direction of association was inferred from $\beta$ value.

\section{Mendelian randomization}

In order to investigate whether DNA methylation is a cause or a consequence of differential gene expression, Mendelian randomization (MR) test was performed using the significant DMR-gene expression associations. Only the associations where the DMR and the gene are within $1 \mathrm{Mb}$ of each other were used. In the model 
where DMR is a cause, we identify instrumental SNPs that are significantly associated with DNA methylation, but not associated with gene expression. All the significant SNPs within $1 \mathrm{Mb}$ of DMRs were extracted, and the associations between these SNPs with expression were calculated using the mixed model adjusting for $Q$ and $K$. The SNPs that are significantly associated with gene expression $(P<0.05 /$ DMR number) were filtered out, and the one with the most significant association with DNA methylation from the remaining SNPs was kept. The observed effect of the SNP on expression from the mixed model was then compared with the predicted effect which was calculated using the following formula,

$$
\beta_{\text {pred }}=\beta_{\text {expr } \sim \mathrm{DMR}} \times \beta_{\mathrm{DMR} \sim \mathrm{SNP}}
$$

In the consequential analysis, instrumental SNPs were required to be associated with gene expression but not with DMR. First, all significant SNPs within $1 \mathrm{Mb}$ of genes were extracted. Then, the SNPs with significant associations with DMR $(P<0.05 /$ gene number $)$ were filtered out, and then, the most significant SNP with gene expression was chosen from the remaining SNP list. We then calculated the effect of gene expression on DMR using gene expression as independent variable and DNA methylation as response. The observed effect of SNP on DMR was from the model DMR SNP. And the predicted effect was calculated as follows:

$$
\beta_{\text {pred }}=\beta_{\text {expr } \sim \mathrm{SNP}} \times \beta_{\mathrm{DMR} \sim \operatorname{expr}}
$$

The above analysis was performed separately for each of the three sequence contexts.

\section{Supplementary information}

Supplementary information accompanies this paper at https://doi.org/10. 1186/s13059-019-1859-0.

Additional file 1: Figure S1. DMR validation. Comparison between DNA methylation of the capture based assay and the whole genome bisulfite sequencing assay (WGBS) in DMRs between B73 and Mo17. Figure S2.

Distribution of DMR size and genomic features. Figure S3. DNA

methylation can reflect genetic distances. Figure S4. DNA methylation levels in DMRs are different among maize subgroups. Figure S5. Summary of DMRs associated with SNPs and structure variants (SVs). Figure S6. The associations between gene expression and DNA methylation. Figure $\mathbf{S 7 .}$ DMRs affect gene expression as a cause. Figure $\mathbf{5 8}$. Mendelian randomization analysis. Figure $\mathbf{S 9}$. The association between DMR and metabolic trait chr di-C.

Additional file 2: Table S1. Summary of data generated in this study Table S2. DMRs with significant SNPs. Table S3. Associations between DMR and gene expression in kernel and leaf. Table S4. Associations between DMRs and metabolic traits.

Additional file 3. Review history.

\section{Acknowledgements}

Not applicable

Review history

The review history is available as Additional file 3.

\section{Peer review information}

Kevin Pang was the primary editor on this article and managed its editorial process and peer review in collaboration with the rest of the editorial team.

\section{Authors' contributions}

QL and NMS designed the study. JX, GC, PJH, QX, CS, WC, QK, and ML performed the experiments. JX, QL, GC, QX, and NMS analyzed the data. QL, NMS, and JX wrote the manuscript. JY, LL, and PAC provided critical input to the data analysis and manuscript revisions. All authors read, revised, and approved the manuscript.

\section{Funding}

This research was supported by The National Key Research and Development Program of China (2016YFD0101003), the Thousand Young Talents Program, the Huazhong Agricultural University Scientific \& Technological Self-innovation Foundation (2016RC012), and the Fundamental Research Funds for the Central Universities (2662017PY033). PJH, PC, and NMS are supported by grants from NSF (IOS-1802848) and from the Minnesota Agricultural Experiment Station (MIN 71-068).

\section{Availability of data and materials}

The datasets generated during the current study has been deposited into the National Center for Biotechnology Information under project number PRJNA549173 [74]. The ChIP-seq data for H3K9me2 (SRR1482372) and H3K27me3 (SRR5436222) were downloaded from NCBI [68, 69].

Ethics approval and consent to participate

Not applicable

\section{Consent for publication}

Not applicable

\section{Competing interests}

The authors declare that they have no competing interests.

\section{Author details}

${ }^{1}$ National Key Laboratory of Crop Genetic Improvement, Huazhong Agricultural University, Wuhan 430070, China. ${ }^{2}$ Institute of Nuclear and Biological Technology, Xinjiang Academy of Agricultural Sciences, Urumqi 830091, China. ${ }^{3}$ Department of Plant and Microbial Biology, University of Minnesota, St. Paul, MN 55108, USA.

Received: 4 May 2019 Accepted: 10 October 2019

Published online: 19 November 2019

\section{References}

1. Springer NM, Schmitz RJ. Exploiting induced and natural epigenetic variation for crop improvement. Nat Rev Genet. 2017;18:563-75.

2. Law JA, Jacobsen SE. Establishing, maintaining and modifying DNA methylation patterns in plants and animals. Nat Rev Genet. 2010;11:204-20.

3. Li Q, Eichten SR, Hermanson PJ, Zaunbrecher VM, Song J, Wendt J, Rosenbaum H, Madzima TF, Sloan AE, Huang J, et al. Genetic perturbation of the maize methylome. Plant Cell. 2014;26:4602-16.

4. Ono A, Yamaguchi K, Fukada-Tanaka S, Terada R, Mitsui T, lida S. A null mutation of ROSia for DNA demethylation in rice is not transmittable to progeny. Plant J. 2012;71:564-74.

5. Moritoh S, Eun CH, Ono A, Asao H, Okano Y, Yamaguchi K, Shimatani Z, Koizumi A, Terada R. Targeted disruption of an orthologue of DOMAINS REARRANGED METHYLASE 2, OsDRM2, impairs the growth of rice plants by abnormal DNA methylation. Plant J. 2012;71:85-98.

6. Tan F, Zhou C, Zhou Q, Zhou S, Yang W, Zhao Y, Li G, Zhou DX. Analysis of chromatin regulators reveals specific features of rice DNA methylation pathways. Plant Physiol. 2016;171:2041-54.

7. Zemach A, Kim MY, Hsieh PH, Coleman-Derr D, Eshed-Williams L, Thao K, Harmer SL, Zilberman D. The Arabidopsis nucleosome remodeler DDM1 allows DNA methyltransferases to access $\mathrm{H1}$-containing heterochromatin. Cell. 2013;153:193-205.

8. Stroud H, Do T, Du J, Zhong X, Feng S, Johnson L, Patel DJ, Jacobsen SE. Non-CG methylation patterns shape the epigenetic landscape in Arabidopsis. Nat Struct Mol Biol. 2014;21:64-72. 
9. Schmitz RJ, Schultz MD, Lewsey MG, O'Malley RC, Urich MA, Libiger O, Schork NJ, Ecker JR. Transgenerational epigenetic instability is a source of novel methylation variants. Science. 2011;334:369-73.

10. Becker C, Hagmann J, Muller J, Koenig D, Stegle O, Borgwardt K, Weigel D. Spontaneous epigenetic variation in the Arabidopsis thaliana methylome. Nature. 2011:480:245-9.

11. van der Graaf A, Wardenaar R, Neumann DA, Taudt A, Shaw RG, Jansen RC, Schmitz RJ, Colome-Tatche M, Johannes F. Rate, spectrum, and evolutionary dynamics of spontaneous epimutations. Proc Natl Acad Sci U S A. 2015;112: 6676-81.

12. Schmitz RJ, Schultz MD, Urich MA, Nery JR, Pelizzola M, Libiger O, Alix A, McCosh RB, Chen H, Schork NJ, et al. Patterns of population epigenomic diversity. Nature. 2013;495:193-8.

13. Li Q, Song J, West PT, Zynda G, Eichten SR, Vaughn MW, Springer NM. Examining the causes and consequences of context-specific differential DNA methylation in maize. Plant Physiol. 2015;168:1262-74.

14. Richards EJ. Inherited epigenetic variation — revisiting soft inheritance. Nat Rev Genet. 2006;7:395-401.

15. Luff B, Pawlowski L, Bender J. An inverted repeat triggers cytosine methylation of identical sequences in Arabidopsis. Mol Cell. 1999;3:505-11.

16. Kawakatsu T, Huang SC, Jupe F, Sasaki E, Schmitz RJ, Urich MA, Castanon R, Nery JR, Barragan C, He Y, et al. Epigenomic diversity in a global collection of Arabidopsis thaliana accessions. Cell. 2016;166:492-505.

17. Dubin MJ, Zhang P, Meng D, Remigereau MS, Osborne EJ, Paolo Casale F, Drewe $P$, Kahles A, Jean G, Vilhjalmsson B, et al. DNA methylation in Arabidopsis has a genetic basis and shows evidence of local adaptation. Elife. 2015;4:e05255.

18. Eichten SR, Briskine R, Song J, Li Q, Swanson-Wagner R, Hermanson PJ, Waters AJ, Starr E, West PT, Tiffin P, et al. Epigenetic and genetic influences on DNA methylation variation in maize populations. Plant Cell. 2013;25: 2783-97.

19. Li Q, Eichten SR, Hermanson PJ, Springer NM. Inheritance patterns and stability of DNA methylation variation in maize near-isogenic lines. Genetics. 2014;196:667-76.

20. Ong-Abdullah M, Ordway JM, Jiang N, Ooi SE, Kok SY, Sarpan N, Azimi N, Hashim AT, Ishak Z, Rosli SK, et al. Loss of Karma transposon methylation underlies the mantled somaclonal variant of oil palm. Nature. 2015;525:533-7.

21. Regulski M, Lu Z, Kendall J, Donoghue MT, Reinders J, Llaca V, Deschamps S, Smith A, Levy D, McCombie WR, et al. The maize methylome influences mRNA splice sites and reveals widespread paramutation-like switches guided by small RNA. Genome Res. 2013;23:1651-62.

22. O'Malley RC, Huang SC, Song L, Lewsey MG, Bartlett A, Nery JR, Galli M, Gallavotti A, Ecker JR. Cistrome and epicistrome features shape the regulatory DNA landscape. Cell. 2016;165:1280-92.

23. Meng D, Dubin M, Zhang P, Osborne EJ, Stegle O, Clark RM, Nordborg M. Limited contribution of DNA methylation variation to expression regulation in Arabidopsis thaliana. PLoS Genet. 2016;12:e1006141.

24. Wang X, Wang H, Liu S, Ferjani A, Li J, Yan J, Yang X, Qin F. Genetic variation in ZmVPP1 contributes to drought tolerance in maize seedlings. Nat Genet. 2016:48:1233-41.

25. Mao H, Wang H, Liu S, Li Z, Yang X, Yan J, Li J, Tran LS, Qin F. A transposable element in a NAC gene is associated with drought tolerance in maize seedlings. Nat Commun. 2015;6:8326.

26. Schmiedel BJ, Singh D, Madrigal A, Valdovino-Gonzalez AG, White BM, Zapardiel-Gonzalo J, Ha B, Altay G, Greenbaum JA, McVicker G, et al. Impact of genetic polymorphisms on human immune cell gene expression. Cell. 2018;175:1701-15.

27. Zhou P, Hirsch CN, Briggs SP, Springer NM. Dynamic patterns of gene expression additivity and regulatory variation throughout maize development. Mol Plant. 2019;12:410-25.

28. Li Q, Suzuki M, Wendt J, Patterson N, Eichten SR, Hermanson PJ, Green D, Jeddeloh J, Richmond T, Rosenbaum H, et al. Post-conversion targeted capture of modified cytosines in mammalian and plant genomes. Nucleic Acids Res. 2015;43:e81.

29. Han Z, Crisp PA, Stelpflug S, Kaeppler SM, Li Q, Springer NM. Heritable epigenomic changes to the maize methylome resulting from tissue culture. Genetics. 2018;209:983-95.

30. Li Q, Gent JI, Zynda G, Song J, Makarevitch I, Hirsch CD, Hirsch CN, Dawe RK, Madzima TF, McGinnis KM, et al. RNA-directed DNA methylation enforces boundaries between heterochromatin and euchromatin in the maize genome. Proc Natl Acad Sci U S A. 2015;112:14728-33.
31. Jiao Y, Peluso P, Shi J, Liang T, Stitzer MC, Wang B, Campbell MS, Stein JC, Wei $X$, Chin CS, et al. Improved maize reference genome with singlemolecule technologies. Nature. 2017;546:524-7.

32. Shen $X$, De Jonge J, Forsberg SK, Pettersson ME, Sheng Z, Hennig L, Carlborg O. Natural CMT2 variation is associated with genome-wide methylation changes and temperature seasonality. PLoS Genet. 2014;10: e1004842.

33. Woo HR, Pontes O, Pikaard CS, Richards EJ. VIM1, a methylcytosine-binding protein required for centromeric heterochromatinization. Genes Dev. 2007; 21:267-77.

34. Yang N, Liu J, Gao Q, Gui S, Chen L, Yang L, Huang J, Deng T, Luo J, He L, et al. Genome assembly of a tropical maize inbred line provides insights into structural variation and crop improvement. Nat Genet. 2019;51:1052-9.

35. Du J, Johnson LM, Jacobsen SE, Patel DJ. DNA methylation pathways and their crosstalk with histone methylation. Nat Rev Mol Cell Biol. 2015;16:519-32.

36. Weinhofer I, Hehenberger E, Roszak P, Hennig L, Kohler C. H3K27me3 profiling of the endosperm implies exclusion of polycomb group protein targeting by DNA methylation. PLoS Genet. 2010;6:e1001152.

37. Long JC, Xia AA, Liu JH, Jing JL, Wang YZ, Qi CY, He Y. Decrease in DNA methylation 1 (DDM1) is required for the formation of ${ }^{\mathrm{m}} \mathrm{CHH}$ islands in maize. J Integr Plant Biol. 2018;61:749-64.

38. Wen W, Li D, Li X, Gao Y, Li W, Li H, Liu J, Liu H, Chen W, Luo J, et al. Metabolome-based genome-wide association study of maize kernel leads to novel biochemical insights. Nat Commun. 2014;5:3438.

39. Fu FF, Dawe RK, Gent Jl. Loss of RNA-directed DNA methylation in maize chromomethylase and DDM1-type mucleosome remodeler mutants. Plant Cell. 2018;30:1617-27.

40. Hu L, Li N, Xu C, Zhong S, Lin X, Yang J, Zhou T, Yuliang A, Wu Y, Chen Y-R, et al. Mutation of a major CG methylase in rice causes genome-wide hypomethylation, dysregulated genome expression, and seedling lethality. Proc Natl Acad Sci U S A. 2014;111:10642-7.

41. Gent Jl, Ellis NA, Guo L, Harkess AE, Yao Y, Zhang X, Dawe RK. CHH islands: de novo DNA methylation in near-gene chromatin regulation in maize. Genome Res. 2013;23:628-37.

42. Anderson SN, Zynda GJ, Song J, Han Z, Vaughn MW, Li Q, Springer NM. Subtle perturbations of the maize methylome reveal genes and transposons silenced by chromomethylase or RNA-directed DNA methylation pathways. G3. 2018:8:1921-32.

43. Lang Z, Wang Y, Tang K, Tang D, Datsenka T, Cheng J, Zhang Y, Handa AK, Zhu JK. Critical roles of DNA demethylation in the activation of ripeninginduced genes and inhibition of ripening-repressed genes in tomato fruit. Proc Natl Acad Sci U S A. 2017;114:E4511-E9.

44. Lindroth AM, Cao X, Jackson JP, Zilberman D, McCallum CM, Henikoff S, Jacobsen SE. Requirement of CHROMOMETHYLASE3 for maintenance of CpXpG methylation. Science. 2001;292:2077-80.

45. Secco D, Wang C, Shou H, Schultz MD, Chiarenza S, Nussaume L, Ecker JR Whelan J, Lister R. Stress induced gene expression drives transient DNA methylation changes at adjacent repetitive elements. Elife. 2015;4:e09343.

46. Harris CJ, Scheibe M, Wongpalee SP, Liu W, Cornett EM, Vaughan RM, Li X Chen W, Xue Y, Zhong Z, et al. A DNA methylation reader complex that enhances gene transcription. Science. 2018;362:1182-6.

47. Li H, Peng Z, Yang X, Wang W, Fu J, Wang J, Han Y, Chai Y, Guo T, Yang N, et al. Genome-wide association study dissects the genetic architecture of oil biosynthesis in maize kernels. Nat Genet. 2013;45:43-50.

48. Wang H, Xu S, Fan Y, Liu N, Zhan W, Liu H, Xiao Y, Li K, Pan Q, Li W, et al. Beyond pathways: genetic dissection of tocopherol content in maize kernels by combining linkage and association analyses. Plant Biotechnol J. 2018;16:1464-75.

49. Kump KL, Bradbury PJ, Wisser RJ, Buckler ES, Belcher AR, Oropeza-Rosas MA, Zwonitzer JC, Kresovich S, McMullen MD, Ware D, et al. Genome-wide association study of quantitative resistance to southern leaf blight in the maize nested association mapping population. Nat Genet. 2011;43:163-8.

50. Quadrana L, Colot V. Plant transgenerational epigenetics. Annu Rev Genet. 2016:50:467-91.

51. Cortijo S, Wardenaar R, Colome-Tatche M, Gilly A, Etcheverry M, Labadie K, Caillieux E, Hospital F, Aury JM, Wincker P, et al. Mapping the epigenetic basis of complex traits. Science. 2014;343:1145-8.

52. Furci $L$, Jain $R$, Stassen J, Berkowitz $O$, Whelan J, Roquis D, Baillet $V$, Colot $V$, Johannes F, Ton J. Identification and characterisation of hypomethylated DNA loci controlling quantitative resistance in Arabidopsis. Elife. 2019:8:e40655. 
53. Yang X, Gao S, Xu S, Zhang Z, Prasanna BM, Li L, Li J, Yan J. Characterization of a global germplasm collection and its potential utilization for analysis of complex quantitative traits in maize. Mol Breed. 2010;28:511-26.

54. Gao S, Zou D, Mao L, Liu H, Song P, Chen Y, Zhao S, Gao C, Li X, Gao Z, et al. BS-SNPer: SNP calling in bisulfite-seq data. Bioinformatics. 2015;31: 4006-8.

55. Liu H, Luo X, Niu L, Xiao Y, Chen L, Liu J, Wang X, Jin M, Li W, Zhang Q, et al. Distant eQTLs and non-coding sequences play critical roles in regulating gene expression and quantitative trait variation in maize. Mol Plant. 2017:10:414-26.

56. Information of the capture regions. doi: https://doi.org/10.13020/D69X0H.

57. Trim Galore. http://www.bioinformatics.babraham.ac.uk/projects/trim_galore/. Accessed 25 Apr 2017.

58. Xi Y, Li W. BSMAP: whole genome bisulfite sequence MAPping program. BMC Bioinformatics. 2009:10:232.

59. Picard Tools. https://broadinstitute.github.io/picard/. Accessed 26 Apr 2017.

60. Barnett DW, Garrison EK, Quinlan AR, Stromberg MP, Marth GT. BamTools: a $\mathrm{C}++\mathrm{API}$ and toolkit for analyzing and managing BAM files. Bioinformatics. 2011:27:1691-2.

61. Quinlan AR, Hall IM. BEDTools: a flexible suite of utilities for comparing genomic features. Bioinformatics. 2010;26:841-2.

62. Stacklies W, Redestig H, Scholz M, Walther D, Selbig J. pcaMethods-a bioconductor package providing PCA methods for incomplete data. Bioinformatics. 2007;23:1164-7.

63. Yang J, Lee SH, Goddard ME, Visscher PM. GCTA: a tool for genome-wide complex trait analysis. Am J Hum Genet. 2011;88:76-82.

64. Zhang F, Chen W, Zhu Z, Zhang Q, Nabais MF, Qi T, Deary IJ, Wray NR, Visscher PM, McRae AF, et al. OSCA: a tool for omic-data-based complex trait analysis. Genome Biol. 2019;20:107.

65. Kang HM, Sul JH, Service SK, Zaitlen NA, Kong SY, Freimer NB, Sabatti C, Eskin E. Variance component model to account for sample structure in genome-wide association studies. Nat Genet. 2010;42:348-54.

66. Alexander DH, Novembre J, Lange K. Fast model-based estimation of ancestry in unrelated individuals. Genome Res. 2009;19:1655-64.

67. Barrett JC, Fry B, Maller J, Daly MJ. Haploview: analysis and visualization of LD and haplotype maps. Bioinformatics. 2005;21:263-5.

68. West PT, Li Q, Ji L, Eichten SR, Song J, Vaughn MW, Schmitz RJ, Springer NM. Genomic distribution of H3K9me2 and DNA methylation in a maize genome. PLoS One. 2014;9:e105267.

69. Zhao H, Zhang W, Chen L, Wang L, Marand AP, Wu Y, Jiang J. Proliferation of regulatory DNA elements derived from transposable elements in the maize genome. Plant Physiol. 2018;176:2789-803.

70. Langmead B, Salzberg SL. Fast gapped-read alignment with Bowtie 2. Nat Methods. 2012:9:357-9.

71. Ramirez F, Dundar F, Diehl S, Gruning BA, Manke T. deepTools: a flexible platform for exploring deep-sequencing data. Nucleic Acids Res. 2014;42: W187-91.

72. Fu J, Cheng Y, Linghu J, Yang X, Kang L, Zhang Z, Zhang J, He C, Du X, Peng $Z$, et al. RNA sequencing reveals the complex regulatory network in the maize kernel. Nat Commun. 2013;4:2832

73. Kim D, Pertea G, Trapnell C, Pimentel H, Kelley R, Salzberg SL. TopHat2 accurate alignment of transcriptomes in the presence of insertions, deletions and gene fusions. Genome Biol. 2013;14:R36.

74. Xu J, Chen G, Hermanson PJ, Xu Q, Sun C, Chen W, Kan Q, Li M, Crisp PA, Yan J, Li L, Springer NM, Li Q. Population-level analysis reveals the widespread occurrence and phenotypic consequence of DNA methylation variation not tagged by genetic variation. DNA methylation data, NCBI: BioProject: PRJNA549173. https://www.ncbi.nlm.nih.gov/bioproject/PRJNA54 9173 (2019). Accessed 20 Sept 2019

\section{Publisher's Note}

Springer Nature remains neutral with regard to jurisdictional claims in published maps and institutional affiliations.

Ready to submit your research? Choose BMC and benefit from:

- fast, convenient online submission

- thorough peer review by experienced researchers in your field

- rapid publication on acceptance

- support for research data, including large and complex data types

- gold Open Access which fosters wider collaboration and increased citations

- maximum visibility for your research: over $100 \mathrm{M}$ website views per year

At BMC, research is always in progress.

Learn more biomedcentral.com/submissions 